\title{
Large-scale surveillance study of the safety and effectiveness of entecavir in Korean patients with chronic hepatitis $B$
}

\author{
Chang Wook Kim ${ }^{1}$ Chang Seop Kim², Hee Yeon Kim ${ }^{2}$ Chang Don Lee ${ }^{1}$, Kyungha Yu³ Cyril Llamoso ${ }^{4}$, \\ and Heon Ju Lee
}

\begin{abstract}
${ }^{1}$ Department of Internal Medicine, College of Medicine, The Catholic University of Korea, Seoul; ${ }^{2}$ Neane Clinic, Seoul; ${ }^{3}$ Bristol-Myers Squibb, Seoul, Korea; ${ }^{4}$ Bristol-Myers Squibb, Wallingford, CT, USA; ${ }^{5}$ Department of Internal Medicine, Yeungnam University College of Medicine, Daegu, Korea
\end{abstract}

Received: April 4, 2016 Revised : September 3, 2016 Accepted: October 15, 2016

\section{Correspondence to}

Heon Ju Lee, M.D.

Department of Internal Medicine, Yeungnam University College of Medicine, 170 Hyeonchung-ro, Nam-gu, Daegu 42415, Korea

Tel: $+82-53-623-8000$

Fax: $+82-53-654-8386$

E-mail: heonjulee@yu.ac.kr
Background/Aims: Entecavir (ETV) is effective and safe antiviral agent against hepatitis B virus (HBV) in clinical and real-world setting but, most studies were performed in single institute or have limitation in patient's number. A large-scale nation-wide real-world surveillance study was carried out to investigate safety, efficacy and clinical effectiveness of ETV in Korean patients with chronic hepatitis $\mathrm{B}(\mathrm{CHB})$.

Methods: Between 2006 and 2012, 3,444 patients were enrolled from 132 Korean institutions. For the safety assessment, investigators recorded the occurrence of observed and patient-reported adverse events (AEs), as well as laboratory abnormalities. Efficacy, which consisted of change in HBV DNA and alanine aminotransferase (ALT), was evaluated in patients who had received at least 16 weeks of ETV treatment. Overall clinical effectiveness, based on improvement of ALT, HBV DNA and patient's symptoms, was evaluated by physicians.

Results: Of the patients, 3,367 were evaluated for safety and 3,115 for efficacy and clinical effectiveness. Three hundred and eighty AEs were reported in 255 cases (7.57\%), and 67 adverse drug reactions in 54 cases (1.6\%). Serious AEs (SAE) were 19 events in nine cases $(0.27 \%)$. Serious adverse drug reactions (SADR) were three events in two cases (0.06\%), and unexpected SAE/SADR were three events in two cases (0.06\%). Medical history and concomitant medications were factors influencing incidence rates of AEs. Overall clinical effectiveness rate was $96.53 \%$, which was clinically assessed as marked improved or improved state.

Conclusions: This study showed that ETV was well tolerated and clinically effective in Korean patients with CHB in a real-world nation-wide setting.

Keywords: Drug-related side effects and adverse reactions; Product surveillance, postmarketing; Entecavir; Hepatitis B, chronic

\section{INTRODUCTION}

Worldwide, more than 240 million people are living with chronic liver infections, and about 600,000 people die every year due to the acute or chronic consequences of hepatitis B virus (HBV) infections [1]. HBV infection is a major cause of acute and chronic hepatitis, cirrhosis, and hepatocelluar carcinoma (HCC), as well as a factor contributing to death from liver disease in Korea $[2,3]$.

Most Korean chronic hepatitis B (CHB) patients are 
infected with the HBV subgenotype $\mathrm{C} 2$, which is known to have a lower hepatitis $\mathrm{B}$ e-antigen seroconversion rate, more rapid progression to cirrhosis and HCC, a lower interferon treatment response rate, and a higher relapse rate after antiviral treatment, compared with other HBV genotypes [4,5].

Entecavir (ETV) is a cyclopentyl guanosine analog with potent selective inhibition of the priming, DNA-dependent synthesis and reverse transcription functions of the HBV polymerase. Clinical studies have shown it to be a potent, safe, and tolerable antiviral agent, and it is recommended as first-line monotherapy for patients with compensated or decompensated CHB [6]. In real-world cohort studies of more than 1,000 patients treated with ETV for up to 6 years, ETV has shown long-term effectiveness, as well as low rates for development of resistance, and a good safety profile [7]. But, most studies were performed in single or small number of institutes or have limitation in patient's number.

We conducted a large-scale, nation-wide surveillance study in accordance with the Korean Ministry of Food and Drug Safety's protocol for reexamination of new drugs postapproval. The study included 3,444 CHB patients, and was conducted to identify occurrence of all adverse events (AEs), including unexpected adverse drug events and serious adverse events (SAEs) regardless of any causal relationship to the study drug, as well as the factors related to the safety and efficacy of ETV. Furthermore, we investigated all AEs, including unexpected and rare AEs, which had no established relationship with ETV treatment, and proposed alternative explanations for their occurrence, based on closely monitoring these AEs.

Here we report data from this study with regard to the safety and clinical effectiveness of ETV treatment in Korean patients with CHB including liver cirrhosis.

\section{METHODS}

This surveillance study was conducted prospectively in accordance with a protocol approved by the Korean Ministry of Food and Drug Safety (MFDS). Each institution participating in this study received Institutional Review Board (IRB) review for the research approval. approval (for example, IRB no. of Uijeongbu St. Mary's
Hospital UCMCo8MPoo7). Written informed consent was obtained from each patient at the time of enrollment.

\section{Patients}

A total of 3,444 patients were enrolled between May 2006 and May 2012 from 132 Korean institutions. Of these, 39 patients were excluded because they were enrolled prior to contract execution with the institutions, 20 patients had duplicate enrollments, 14 patients were lost to follow-up, three patients had off-label use, and one patient was not administered the study drug, leaving 3,367 patients for safety evaluation. Among these 3,367 patients, 238 were treated for fewer than 16 weeks, and efficacy or clinical effectiveness could not be assessed for a further 14 patients; therefore, 3,115 patients were evaluated for efficacy and clinical effectiveness (Fig. 1).

\section{Data collection}

The mean treatment duration was $258.34 \pm 151.49$ days (median, 204). Two hundred and thirty-one patients (6.88\%) were treated for less than 16 weeks, 793 patients (23.61\%) for ' 16 weeks or more and less than 24 weeks' and 2,335 patients (69.51\%) for more than 24 weeks. During the study period, reported AEs were presented in accordance with the 2010 World Health Organization Adverse Reaction Terminology criteria, with System Organ Class and Preferred Term. For the safety evaluation, an $\mathrm{AE}$ was defined as any event that was not present at the onset of ETV therapy.

Efficacy was evaluated by measuring a change in aspartate aminotransferase (AST), alanine aminotransferase (ALT), HBV DNA, albumin and total bilirubin levels from baseline in patients treated with ETV for at least 16 weeks. Patient demographics, disease information, drug administration reports, occurrence of AEs, and clinical laboratory evaluations were also collected. Changes in ALT levels for biochemical efficacy and HBV DNA levels for virological efficacy were assessed.

Clinical effectiveness data were collected and defined with a 5-level assessment tool (markedly improved, improved, no change, aggravated, and unassessable, compared with 'baseline') according to the physician's assessment, based on ALT, HBV DNA levels and improvement of patient's symptoms. 


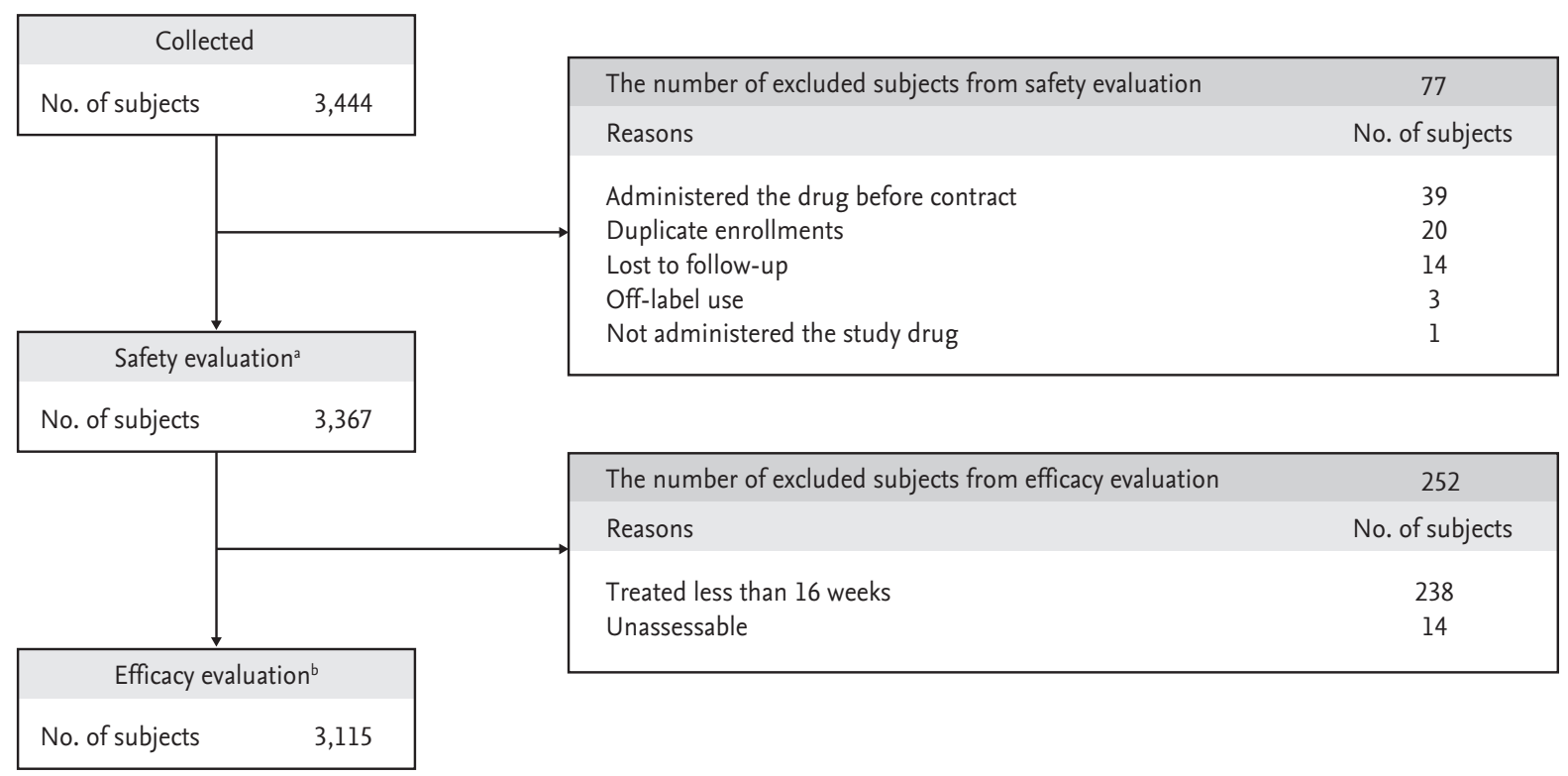

Figure 1. Flow chart of subjects enrolled in this surveillance study. ${ }^{a}$ Entecavir used $\geq$ once, ${ }^{b}$ Entecavir treatment $\geq 16$ weeks.

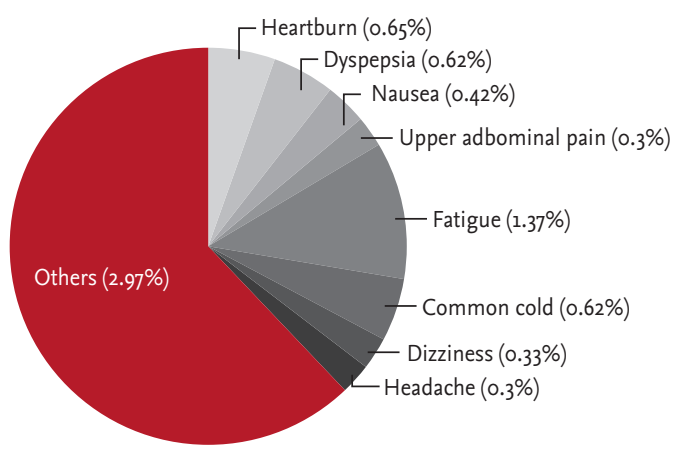

Figure 2. Frequency diagram of adverse events. The most common events were fatigue (1.37\%, 46 events), followed by heartburn (0.65\%, 22 events), dyspepsia (0.62\%, 21 events), nausea $(0.42 \%, 15$ events), and upper abdominal pain $(0.30 \%$, 10 events).

\section{Statistical analysis}

Data are expressed as mean \pm standard deviation (SD) values, or as frequencies and percentages. Statistical analyses were performed using SAS version 9.3 (SAS Institute Inc., Cary, NC, USA). Student $t$ test, paired t test, Pearson chi-squared test, Fisher exact test, and logistic regression analyses were used where appropriate, and performed two-sided at the $5 \%$ significance level. Factors affecting safety and efficacy rates were identified by multivariate analysis using a logistic regression model.

\section{Subgroup analysis}

A post hoc subgroup analysis was carried out on data from 3,444 patients with or without cirrhosis, and duration of ETV therapy was examined. This subgroup was further divided based on medical history, value of baseline platelet count $\left(\leq 100,000 / \mathrm{mm}^{3}\right)$, or medical records on diagnosis of cirrhosis. Patients with unclear information about their cirrhosis status, or those that had never been treated with ETV were excluded from this analysis.

\section{RESULTS}

\section{Baseline characteristics of study patients}

The main baseline characteristics of patients studied for the safety evaluation are shown in Table 1 . There were 2,375 male patients (70.56\%) and 991 female patients (29.44\%), with a mean \pm SD age of $43.74 \pm 11.41$ years. The most populated group was the 40 - to 49 -year-old, which comprised 1,161 patients (34.49\%), followed by the 30- to 39-year-old, comprising 840 patients (24.96\%) and the 50- to 59-year-old with 683 patients (20.29\%). There were also 20 pediatric patients ( $\geq 12$ and $\leq 19$ years old, $0.59 \%$ ), and 139 elderly patients (> 65 years old, 4.13\%).

Most of the study participants were outpatients $(2,821$ patients, $83.78 \%$ ). There were 427 patients with a previ- 
ous medical history (12.68\%), and 476 patients (14.14\%) had concurrent disease, which included 115 patients (3.42\%) with renal disorders, 868 smokers (25.79\%), 1,206 patients (35.82\%) who were alcohol users, and 1,199 patients (35.46\%) with cirrhosis versus 2,182 patients (64.54\%) without cirrhosis.

\section{Other patient characteristics}

Mean period of disease, from diagnosed date to first treatment date with ETV, was $2.92 \pm 4.38$ years. The number of patients who had a family history of CHB $(1,334$ patients, 39.86\%) and this was the most dominant group. The most common routes of infection were through perinatal infection (1,323 patients, 39.32\%), contact with an infected person (627 patients, 18.63\%), and through blood transfusions (17 patients, $0.51 \%)$.

A total of 736 patients $(21.86 \%)$ were taking concomitant medications, the most common being alimentary tract and metabolism class of drugs, which were administered in 522 patients (70.92\%). Other concurrent medications administered were for liver therapy and lipotropics, bile therapy, peptic ulcer and gastroesophageal reflux disease, as well as blood glucose lowering drugs. Cardiovascular medications were administered in 258 patients (35.05\%).

\section{Summary of AEs}

Investigators recorded the occurrence of observed and patient-reported AEs for the safety assessment. Of the patients included in the safety evaluation (Table 2), 380 AEs were reported in 255 (7.57\%) cases. Of these, 67 adverse drug reactions (ADR) were reported in 54 (1.60\%) cases, which may have occurred due to causality with ETV. Unexpected AEs were 253 events in 188 cases (5.58\%), and serious AEs (SAE) were 19 events in nine cases (0.27\%). Serious adverse drug reactions (SADR) were three events

Table 1. Baseline characteristics of study subjects

\begin{tabular}{|c|c|c|}
\hline Characteristic & Mean \pm SD & No. of patients (\%) \\
\hline Male sex & & $2,375(70.56)$ \\
\hline Age, yr (range) & $43.74 \pm 11.41(16-85)$ & \\
\hline Inpatient ${ }^{\mathrm{a}}$ :outpatient & & $546(16.22): 2,821(83.78)$ \\
\hline Medical history (past) & & $427(12.68)$ \\
\hline Concurrent disease & & $476(14.14)$ \\
\hline Renal disorder & & $115(3.42)$ \\
\hline Smoking & & $868(25 \cdot 79)$ \\
\hline Alcohol consumption & & $1,206(35.82)$ \\
\hline Liver cirrhosis & & $1,199(35 \cdot 46)$ \\
\hline Hepatitis B virus DNA, $\log _{10}$ copies/mL & $7.02 \pm 1.76$ & 2,696 \\
\hline Alanine aminotransferase, U/L & $177.93 \pm 265.94$ & 3,103 \\
\hline Aspartate aminotransferase, U/L & $154.63 \pm 217.52$ & 3,101 \\
\hline Entecavir therapy duration, day & $258.34 \pm 151.49$ & 3,359 \\
\hline
\end{tabular}

${ }^{\mathrm{a}}$ Defined as patients have experience with hospitalization more than once.

Table 2. Summary of adverse events

\begin{tabular}{lccc}
\hline Variable & No. of patients & No. of events & Rate (\% of patients) \\
\hline Adverse event & 255 & 380 & 7.57 \\
Adverse drug reaction & 54 & 67 & 1.60 \\
Serious adverse event $_{\text {Serious adverse drug reaction }}{ }^{\mathrm{a}}$ & 9 & 19 & 0.27 \\
Suspected unexpected serious adverse reaction $^{\mathrm{a}}$ & 2 & 3 & 0.06 \\
\hline
\end{tabular}

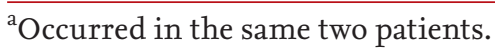


Table 3. Adverse events ${ }^{\mathrm{a}}$ and adverse drug reactions

\begin{tabular}{|c|c|c|c|c|}
\hline \multirow{2}{*}{ Category } & \multicolumn{2}{|c|}{ Adverse events } & \multicolumn{2}{|c|}{ Adverse drug reactions } \\
\hline & No. of cases (\%) & No. of events & No. of cases (\%) & No. of events \\
\hline Gastrointestinal disorder & $104(3.09)$ & 120 & $27(0.8)$ & 30 \\
\hline Heartburn & $22(0.65)$ & 22 & $7(0.21)$ & 8 \\
\hline Dyspepsia & $21(0.62)$ & 21 & $6(0.18)$ & 6 \\
\hline Nausea & $14(0.42)$ & 15 & $4(0.12)$ & 4 \\
\hline Upper abdominal pain & $10(0.30)$ & 10 & $8(0.24)$ & 8 \\
\hline General disorder & $70(2.08)$ & 76 & $12(0.36)$ & 12 \\
\hline Fatigue & $46(1.37)$ & 46 & $10(0.3)$ & 10 \\
\hline Respiratory disorder & $40(1.19)$ & 51 & o & 0 \\
\hline Common cold & $21(0.62)$ & 22 & o & o \\
\hline Cough & $8(0.24)$ & 8 & 0 & o \\
\hline Central \& peripheral nervous system disorder & $26(0.77)$ & 27 & $6(0.18)$ & 6 \\
\hline Dizziness & $11(0.33)$ & 11 & $3(0.09)$ & 3 \\
\hline Headache & $10(0.3)$ & 10 & $3(0.09)$ & 3 \\
\hline Skin and appendages disorder & $15(0.45)$ & 16 & $4(0.12)$ & 4 \\
\hline Pruritus & $7(0.21)$ & 7 & $3(0.09)$ & 3 \\
\hline Musculoskeletal disorder & $14(0.42)$ & 15 & o & o \\
\hline Urinary tract disorder & $10(0.3)$ & 10 & $3(0.09)$ & 3 \\
\hline Neoplasm & $9(0.27)$ & 10 & $\circ$ & o \\
\hline Hepatic neoplasm & $8(0.24)$ & 9 & o & o \\
\hline Others & $100(2.97)$ & 171 & o & o \\
\hline Total & $255(7.57)$ & 378 & $53(1.57)$ & 65 \\
\hline
\end{tabular}

${ }^{\mathrm{a}}$ Adverse events occurred in $\geq 0.2 \%$ of patients.

Table 4. Serious adverse events

\begin{tabular}{lcccc}
\hline Adverse event $^{\text {a }}$ & Severity & Action taken & Outcome & Relationship \\
\hline Abdominal pain & Mild & None & Improved & Probably not \\
Nasal septum deviation & NA & NA & Resolved & Probably not \\
Sinusitis & NA & NA & Resolved & Probably not \\
Eyelid skin disorder & NA & NA & NA & NA \\
Hepatic neoplasm & Severe & None & Unable to determine & Probably not \\
Hepatic neoplasm & Moderate & None & No change & Probably not \\
Hepatic neoplasm & Severe & None & Improved & Probably not \\
Hepatic neoplasm & Moderate & None & Unable to determine & Probably not \\
Hepatic neoplasm & Severe & None & Resolved & Probably not \\
Gastrointestinal hemorrhage & Moderate & None & Resolved & Probably not \\
Gastric angiodysplasia & NA & NA & Resolved & Unable to determine \\
Portal hypertension & NA & NA & Resolved & Unable to determine \\
(portal hypertensive gastropathy) & & & Aggravated & Probably not \\
Cholangitis & Severe & None & Aggravated & Probably not \\
Sepsis & Severe & None & Improved & Probably not \\
Ascites & Moderate & None & Improved & Probably not \\
\hline Ascites & Moderate & None & &
\end{tabular}


Table 4. Continued

\begin{tabular}{lcccc}
\hline Adverse event $^{\text {a }}$ & Severity & Action taken & Outcome & Relationship \\
\hline Hemoperitoneum & Moderate & None & Improved & Probably not \\
Hemoperitoneum & Moderate & None & Improved & Probably not \\
Hepatic encephalopathy & Severe & None & Resolved & Probably not \\
\hline
\end{tabular}

NA, not available.

${ }^{a}$ Based on the World Health Organization Adverse Reactions Terminology criteria.

in two cases (0.06\%), and unexpected SAE/SADR were three events in two cases (0.06\%). SADRs were in the same patients. SADRs and suspected, unexpected, serious adverse reactions are also shown in Table 2, namely eyelid skin disorder, gastric angiodysplasia, and portal hypertensive gastropathy.

Looking at all the AEs over this study (Table 3), regardless of the causal relationship with ETV therapy, gastrointestinal disorders were the most frequent (3.09\%, 104 cases with 120 events). For those cases where a specific event was reported, the most common events were heartburn (0.65\%, 22 events), dyspepsia (0.62\%, 21 events), nausea (0.42\%, 15 events), and upper abdominal pain (0.30\%, 10 events) (Fig. 2).

Unexpected AEs and ADRs associated with the gastrointestinal system, such as heartburn (0.65\%, 23 events) and upper abdominal pain (0.30\%, 10 events) were commonly observed.

SAEs are shown in Table 4. The severity of SAEs reported ranged from mild to severe, and action taken with ETV therapy was 'none' in most SAEs.

\section{Predictive factors by multivariate analysis}

Logistic regression analyses were performed to identify the factors that were suspected to have influenced the reported 380 AEs in 255 patients. For the safety analysis, the incidence of AEs was classified as the dependent variable while several factors derived from the case report forms were considered as independent variables, including gender, age, in/outpatient, medical history, concurrent disease, smoking, alcoholic history, family history, severity prior medication, daily dose, treatment duration, total treatment dose, concomitant medication, and renal disease (Table 5). The multivariate analysis (Table 5) revealed that gender, in/outpatient, medical history, family history, treatment duration, total treatment dose, and concomitant medication had influenced incidence rates for the reported AEs. Specifically, an AE was less likely to occur in males (odds ratio [OR], o.61; 95\% confidence interval [CI], 0.45 to $0.83 ; p=0.002$ ), patients undergoing long treatment durations (OR, 0.68 ; 95\% CI, 0.53 to $0.88 ; p=0.003)$, and more likely to occur in inpatients (OR, 1.77; 95\% CI, 1.28 to $2.44 ; p=0.001$ ), patients with a medical history (OR, 1.94; 95\% CI, 1.39 to 2.70; $p<0.000$ ), patients with a family history of disease (OR, 1.33; 95\% CI, 1.00 to 1.76; $p=0.047$ ), patients receiving > $115 \mathrm{mg}$ treatment dose in total (OR, 1.46; 95\% CI, 1.02 to 2.09 ; $p=0.038)$, and patients receiving concomitant medications (OR, 3.84; 95\% CI, 2.81 to 5.24 ; $p<0.000$ ).

Similarly, the logistic regression analysis was conducted with efficacy as the dependent variable and independent variables from the patient case reports (Table 6). The analysis revealed that prior antiviral medication was the only risk factor contributing to poor efficacy for ETV therapy and, therefore, therapy was more effective in patients without prior antiviral medication (OR, 0.44 ; $95 \%$ CI, 0.27 to $0.71 ; p=0.001$ ).

\section{Efficacy and clinical effectiveness evaluation}

Table 7 shows the change in AST, ALT, HBV DNA, albumin, and total bilirubin before and after ETV treatment. Mean ALT levels before and after ETV treatment were $177.93 \pm 265.94$ and $34.71 \pm 60.38 \mathrm{IU} / \mathrm{L}$, respectively, and mean decrease in ALT was 143.21 $\pm 274.09 \mathrm{IU} / \mathrm{L}(p<$ 0.000). Mean AST before and after treatment were 154.63 \pm 217.52 and $32.85 \pm 24.54 \mathrm{IU} / \mathrm{L}$, respectively, and mean decrease in AST was $121.78 \pm 219.68 \mathrm{IU} / \mathrm{L}(p<0.000)$. Mean HBV DNA before and after treatment were $7.02 \pm 1.76$ and $3.79 \pm 1.79 \log _{10}$ copies/mL, respectively, and mean decrease in HBV DNA was $3.23 \pm 1.92 \log _{10}$ copies $/ \mathrm{mL}$ $(p<0.000)$. Mean albumin before and after ETV treatment were $4.07 \pm 0.57$ and $4.22 \pm 0.49 \mathrm{~g} / \mathrm{dL}$, respectively, and mean increase in albumin was $0.16 \pm 0.44 \mathrm{~g} / \mathrm{dL}(p<$ 
0.000). Mean total bilirubin before and after ETV treatment were $1.28 \pm 1.55$ and $0.98 \pm 0.68 \mathrm{mg} / \mathrm{dL}$, respectively, and mean decrease in total bilirubin was $0.30 \pm 1.52 \mathrm{mg} /$ $\mathrm{dL}(p<0.000)$. Although the mean treatment duration was relatively short, but 258 days, these laboratory parameters were improved significantly.

We also analysed the overall clinical effectiveness rate and efficacy-related factors regarding cases that were

Table 5. Factors associated with occurrence of adverse events (logistic regression)

\begin{tabular}{|c|c|c|c|c|c|}
\hline Factor & Estimate & SE & OR & $95 \% \mathrm{CI}$ & $p$ value \\
\hline Sex (male vs. female) & -0.50 & 0.16 & 0.61 & $0.45-0.83$ & $0.002^{\mathrm{a}}$ \\
\hline Age, yr & 0.00 & 0.01 & 1.00 & $0.98-1.01$ & 0.628 \\
\hline Patient type (inpatient vs. outpatient) & 0.57 & 0.16 & 1.77 & $1.28-2.44$ & $0.001^{\mathrm{a}}$ \\
\hline Medical history (yes vs. no) & 0.66 & 0.17 & 1.94 & $1.39-2.70$ & $<0.000^{\mathrm{a}}$ \\
\hline Concurrent disease (yes vs. no) & 0.25 & 0.19 & 1.28 & $0.88-1.86$ & 0.192 \\
\hline Smoker (yes vs. no) & -0.25 & 0.19 & 0.78 & $0.54-1.13$ & 0.188 \\
\hline Alcohol use (yes vs. no) & 0.27 & 0.17 & 1.32 & $0.95-1.83$ & 0.100 \\
\hline Family history (yes vs. no) & 0.29 & 0.14 & 1.33 & $1.00-1.76$ & $0.047^{\mathrm{a}}$ \\
\hline Severity & 0.04 & 0.11 & 1.04 & $0.84-1.30$ & 0.706 \\
\hline Prior medications (yes vs. no) & 0.07 & 0.17 & 1.07 & $0.77-1.49$ & 0.691 \\
\hline Daily dose, $\mathrm{mg}(>0.5$ vs. $\leq 0.5)$ & -0.34 & 0.24 & 0.71 & $0.44-1.15$ & 0.164 \\
\hline Treatment duration, wk (> 24 vs. $\leq 24$ ) & -0.38 & 0.13 & 0.68 & $0.53-0.88$ & $0.003^{\mathrm{a}}$ \\
\hline Total treatment dose, mg (> 115 vs. $\leq 115)$ & 0.38 & 0.18 & 1.46 & $1.02-2.09$ & $0.038^{\mathrm{a}}$ \\
\hline Concomitant medicine (yes vs. no) & 1.34 & 0.16 & 3.84 & $2.81-5.24$ & $<0.000^{\mathrm{a}}$ \\
\hline Renal disease (yes vs. no) & -0.57 & 0.39 & 0.57 & $0.26-1.21$ & 0.144 \\
\hline
\end{tabular}

SE, standard error; OR, odds ratio; CI, confidence interval.

$\mathrm{a} p<0.005$.

Table 6. Factors associated with entecavir efficacy (logistic regression)

\begin{tabular}{|c|c|c|c|c|c|}
\hline Factor & Estimate & SE & OR & $95 \% \mathrm{CI}$ & $p$ value \\
\hline Sex (male vs. female) & -0.51 & 0.27 & 0.60 & $0.36-1.01$ & 0.056 \\
\hline Age, yr & -0.01 & 0.01 & 0.99 & $0.97-1.01$ & 0.180 \\
\hline Patient type (inpatient vs. outpatient) & 0.42 & 0.31 & 1.52 & $0.83-2.79$ & 0.178 \\
\hline Medical history (yes vs. no) & -0.19 & 0.28 & 0.83 & $0.48-1.45$ & 0.513 \\
\hline Concurrent disease (yes vs. no) & 0.24 & 0.36 & 1.27 & $0.63-2.57$ & 0.498 \\
\hline Smoker (yes vs. no) & 0.17 & 0.26 & 1.19 & $0.72-1.97$ & 0.495 \\
\hline Alcohol use (yes vs. no) & -0.11 & 0.23 & 0.90 & $0.57-1.40$ & 0.629 \\
\hline Family history (yes vs. no) & 0.24 & 0.21 & 1.27 & $0.84-1.94$ & 0.257 \\
\hline Severity & -0.27 & 0.16 & 0.76 & $0.56-1.05$ & 0.092 \\
\hline Prior medications (yes vs. no) & -0.82 & 0.25 & 0.44 & $0.27-0.71$ & $0.001^{\mathrm{a}}$ \\
\hline Daily dose, $\mathrm{mg}(>0.5$ vs. $\leq 0.5)$ & -0.50 & 0.29 & 0.60 & $0.34-1.07$ & 0.083 \\
\hline Treatment duration, wk (> 24 vs. $\leq 24$ ) & -0.33 & 0.27 & 0.72 & $0.43^{-1.22}$ & 0.223 \\
\hline Total treatment dose, mg (> 115 vs. $\leq 115)$ & 0.28 & 0.27 & 1.32 & $0.78-2.26$ & 0.303 \\
\hline Concomitant medicine (yes vs. no) & -0.32 & 0.25 & 0.72 & $0.44-1.18$ & 0.193 \\
\hline Renal disease (yes vs. no) & -0.81 & 0.49 & 0.45 & $0.17-1.17$ & 0.101 \\
\hline
\end{tabular}

SE, standard error; OR, odds ratio; CI, confidence interval.

${ }^{\mathrm{a}} \mathrm{p}<0.005$. 
Table 7. Efficacy evaluation: changes in laboratory test markers related to hepatitis

\begin{tabular}{lccccc}
\hline Variable & No. & Baseline & Follow-up & Difference & $p$ value \\
\hline Albumin & 2,329 & $4.07 \pm 0.57$ & $4.22 \pm 0.49$ & $0.16 \pm 0.44$ & $<0.000$ \\
Total bilirubin & 2,533 & $1.28 \pm 1.55$ & $0.98 \pm 0.68$ & $-0.30 \pm 1.52$ & $<0.000$ \\
Alanine aminotransferase & 3,103 & $177.93 \pm 265.94$ & $34.71 \pm 60.38$ & $-143.21 \pm 274.09$ & $<0.000$ \\
Aspartate aminotransferase & 3,101 & $154.63 \pm 217.52$ & $32.85 \pm 24.54$ & $-121.78 \pm 219.68$ & $<0.000$ \\
Hepatitis B virus DNA $^{2}$ & 2,696 & $7.02 \pm 1.76$ & $3.79 \pm 1.79$ & $-3.23 \pm 1.92$ & $<0.000$ \\
\hline
\end{tabular}

Values are presented as mean $\pm \mathrm{SD}$. Paired $t$ test was used for statistical analysis.

${ }^{\mathrm{a}} \log 10$ copies/mL.

Table 8. Overall efficacy evaluation assessed by physician

\begin{tabular}{lc}
\hline Category & No. $(\%)$ \\
\hline Markedly improved & $1,449(46.52)$ \\
Improved & $1,558(50.02)$ \\
No change & $87(2.79)$ \\
Aggravated & $21(0.67)$ \\
Efficacy cases & $3,007(96.53)$ \\
No efficacy cases & $108(3.47)$ \\
\hline
\end{tabular}

Table 9. Subgroup analysis: serious adverse events ${ }^{\mathrm{a}}$ by cirrhosis status

\begin{tabular}{lccc}
\hline $\begin{array}{l}\text { Serious adverse } \\
\text { event }\end{array}$ & Cirrhosis & Non-cirrhosis & p value \\
\hline No & $1,193(99.50)$ & $2,181(99.95)$ & 0.010 \\
Yes & $6(0.50)$ & $1(0.05)$ & \\
\hline
\end{tabular}

Pearson chi-square test and Fisher exact test were used for statistical analyses.

${ }^{a}$ Excludes 63 patients with missing information.

'markedly improved' and 'improved' (Table 8). Of the 3,367 patients evaluated for safety, 3,115 were also evaluated for efficacy and clinical effectiveness (238 patients treated $<16$ weeks and a further 14 patients for whom efficacy or effectiveness could not be assessed were excluded). Of these, the number of patients evaluated as 'markedly improved' and 'improved' were 1,449 (46.52\%) and 1,558 (50.02\%), respectively. A further 87 patients $(2.79 \%)$ were evaluated as having 'no change,' and 21 patients (0.67\%) were evaluated as 'aggravated.' Therefore, overall clinical effectiveness rate, which was assessed by physician who took care of patients, based on ALT, HBV DNA and improvement of symptoms, was $96.53 \%$ (3,007/3,115 patients).

\section{Subgroup analysis}

The analysis showed that $35.46 \%$ of patients had cirrhosis, and that AEs were more common in these patients than in cirrhosis-free patients. AEs in both groups were those more commonly associated with the gastrointestinal system. In total, AEs were reported in 104 patients with cirrhosis (8.67\%), and 142 patients without cirrhosis (6.51\%, $p=0.020)$.

SAEs were 10 events in six patients with cirrhosis (0.50\%), and five events in one patient without cirrhosis (0.05\%) (Table 9). Due to missing information for two patients, data could not be analysed for four events that were reported for those two patients. There was a significant difference in the rates of SAEs in patients with cirrhosis compared with those without $(p=0.010)$. A higher rate of serious hepatic AEs (regardless of causality) has been observed in patients with liver cirrhosis.

\section{DISCUSSION}

The safety profile of ETV in phase III studies involving more than 1,000 patients has been established to be generally well tolerated [8-10]. Carcinogenesis has been observed in animal models exposed to very high doses of ETV [11], giving rise to an ongoing study of clinical outcomes in more than 12,500 patients with $\mathrm{CHB}$ receiving long-term ETV versus other antiviral agents $[12,13]$. However, to date, there is no evidence for the occurrence of cancers as a result of ETV treatment in patients. Mitochondrial toxicity has been raised as a potential concern associated with nucleos(t)ides, as they can inhibit the mitochondrial polymerase-gamma, causing mitochondrial DNA depletion and subsequent mitochondrial toxicity. However, ETV has a low potential for interfering with the mitochondrial polymerase-gamma [14]. A 
consequence of mitochondrial toxicity can be lactic acidosis, which appears to be observed more frequently in patients with decompensated disease. ETV is well tolerated in patients with decompensated cirrhosis; findings from this surveillance did not reveal occurrence of lactic acidosis, consistent with previously published clinical trial data, as well as several other reports of Korean, Chinese, and EU patient cohorts in real-world settings [13,15-17].

The incidence rate of AEs over the surveillance period was $7.57 \%$, and the rate of ADRs (which cannot rule out occurrence due to a causal relationship with the treatment) was $1.60 \%$. Unlike clinical trials, which are conducted under standardized conditions and with strict safety monitoring, some limitation abound in a real clinical practice setting in terms of collection of safety data, thus limiting direct comparisons. When comparing with AE profiles reported in a clinical trial comprising more than 1,000 subjects (ETV 0.5 or $1 \mathrm{mg}$ ), upper respiratory infection, headache and nasopharyngitis were commonly reported AEs, regardless of the causality with ETV treatment [18]. In this surveillance, gastrointestinal disorders were reported as the most common AEs, including upper abdominal pain, heartburn, dyspepsia, and nausea. ADRs were infrequent, and reported for myalgia and neuropathy-related AEs in comparison clinical trials, whilst gastrointestinal disorders were the most common AEs reported in our surveillance.

When evaluating baseline predictive factors of safety outcomes by logistic regression analysis, we found that gender, inpatients, medical history, family history, and concomitant medication were associated with incidence of AEs. Considering that most of the concomitant medications were anti-inflammatory drugs and most AEs were gastrointestinal symptoms, it is surmised that the incidence rate of AEs is likely to be influenced by various factors related to concomitant medications, rather than directly to ETV therapy.

In addition, it is considered that the general health of inpatients and patients with a medical history or on concomitant medications might be weaker than that of outpatients, and patients without a medical history or on concomitant medications. Therefore, factors related to the general health condition of patients could not be excluded for the analysis on incidence rates of AEs.

Our results indicate that gender and family histo- ry also seemed to influence the occurrence of AEs, but the analysis does not allow for conclusive association, and further study and data collection will be required. However, the results did show that safety profiles were similar between treatment-naive and treatment-experienced populations, in line with findings from previous reports [13].

There was statistical difference in the rates of AEs and SAEs for patients with and without cirrhosis; however, this was not considered clinically significant as the number of SAEs was not adequate for comparison (10 events vs. five events, six cases vs. one case for patients with and without cirrhosis, respectively). Consistent with data from large Phase III trials, our study showed that ETV therapy gave rise to few AEs in patients with compensated liver disease $[8,9]$. Treatment was also more effective in patients who had not taken any prior antiviral medications; it is thought that the difference in the efficacy rate is related to the decreased sensitivity to ETV for lamivudine (LAM)-refractory patients. Previous reports have shown that the presence of LAM-resistant mutations was significantly associated with a reduced efficacy of ETV [10]. Our report confirms these previous findings that the antiviral efficacy of ETV was seriously diminished in LAM-refractory patients. Of all the factors considered as having the potential to impact ETV efficacy, there were no clinically remarkable factors identified in this surveillance, although some were shown to be statistically significant.

There are several study limitations that were identified. First, the treatment duration (mean $258.34 \pm 151.49$ days) was not long enough to allow all safety data to be collected and evaluated. Second, resistance profiles were not investigated in this study. However, it is well documented that ETV has a high genetic barrier to resistance and a strong resistance profile, whereby the cumulative probability of genotypic resistance to ETV of more than 5 years of therapy has been shown to be $1.2 \%$ in nucleos(t)ide-naive patients $[19,20]$. Also of note, in the absence of a standard evaluating tool at the time of designing this study, different assays were used for evaluating efficacy (e.g., for measuring HBV DNA levels), which may have caused possible bias between different laboratories. Also, the baseline genotype was not measured in this study, as pretreatment genotyping is not routinely carried out in clinical practice in Korea. However, HBV 
genotype $\mathrm{C}$ is the most prevalent genotype in Korea and patients were most likely infected with HBV genotype C [4]. Finally, there was no control arm to compare safety profiles, or analyze any causal relationship between emerging AEs and relevant influencing factors, or ETV treatment. Despite these limitations, the strength of this study is that it provides reference data for safety events that can occur in nation-wide real practice for a large number of heterogeneous CHB patients, over a 6-year period. A number of real-world studies comprising large numbers of CHB patients have recently been published, but most have focused on efficacy or resistance profiles, not safety profiles. Real-world studies provide valuable information about treatments in clinical practice, as they include patient populations usually under-represented in clinical studies and, thus, can identify rare or late-emerging AEs. In this respect, our surveillance has the unique feature of conducting both safety and efficacy analyses for ETV treatment, based on nation-wide routine clinical practice in Korea.

In addition, we evaluated clinical effectiveness which means improvements of not only laboratory parameters such as ALT and HBV DNA but also patient's subjective well-being sense and symptoms. This effectiveness of ETV was assessed by physician who took care of patients, with a 5-level assessment tool (markedly improved, improved, no change, aggravated, and unassessable, compared with baseline). Clinical effectiveness is important in real practice because it includes improvement of patient's symptoms as well as ALT and HBV DNA. More than $95 \%$ of patients with ETV therapy assessed as marked improved or improved state compared to baseline in our nation-wide surveillance study.

In summary, this large-scale nation-wide surveillance study confirmed that ETV is well tolerated and clinically effective in Korean patients with CHB in a real-world setting. The results suggest that medical history and concomitant medications were factors influencing $\mathrm{AE}$ incidence rates of patients under ETV therapy so, patients with other medical history, or if administered concomitant medicines should be closely monitored.

\section{KEY MESSAGE}

1. Eetecavir is well tolerated and clinically effective in Korean patients with chronic hepatitis B in a real-world setting.

2. Regardless of the causality with entecavir therapy, 'gastrointestinal system disorder' including heartburn, dyspepsia, nausea, and upper abdominal pain was most common adverse event.

3. Concomitant medication was high risk factor influencing adverse event incidence rates of patients under entecavir therapy so, patients with concomitant medicines should be closely monitored.

\section{Conflicts of interest}

This surveillance was sponsored by Bristol-Myers Squibb Korea. Kyungha Yu and Cyril Llamoso are employees of Bristol-Myers Squibb. Others are no conflict of interest relevant to this article.

\section{Acknowledgments}

We thank all 132 institutions and co-investigators who actively participated to this study.

\section{REFERENCES}

1. World Health Organization(WHO). Hepatitis B [Internet]. Geneva: WHO, c2017 [cited 2017 Sep 5]. Available from: http://www.who.int/mediacentre/factsheets/fs204/en/index.html.

2. Chae HB, Kim JH, Kim JK, Yim HJ. Current status of liver diseases in Korea: hepatitis B. Korean J Hepatol 2009;15 Suppl 6:S13-S24.

3. Korea Centers for Disease Control \& Prevention. Korea National Health and Nutrition Examination Survey [Internet]. Cheongju (KR): Korea Centers for Disease Control \& Prevention, c2012 [cited 2017 Sep 5]. Available from: http://knhanes.cdc.go.kr/knhanes/index.do.

4. Bae SH, Yoon SK, Jang JW, et al. Hepatitis B virus genotype $\mathrm{C}$ prevails among chronic carriers of the virus in Korea. J Korean Med Sci 2005;20:816-820.

5. Kim H, Jee YM, Song BC, et al. Molecular epidemiology of hepatitis $\mathrm{B}$ virus (HBV) genotypes and serotypes in patients with chronic HBV infection in Korea. Intervirology 2007;50:52-57.

6. Korean Association for the Study of the Liver. KASL clinical practice guidelines: management of chronic hepatitis 
B. Clin Mol Hepatol 2012;18:109-162.

7. Pol S, Lampertico P. First-line treatment of chronic hepatitis B with entecavir or tenofovir in 'real-life' settings: from clinical trials to clinical practice. J Viral Hepat 2012;19:377-386.

8. Chang TT, Gish RG, de Man R, et al. A comparison of entecavir and lamivudine for HBeAg-positive chronic hepatitis B. N Engl J Med 2006;354:1001-1010.

9. Lai CL, Shouval D, Lok AS, et al. Entecavir versus lamivudine for patients with HBeAg-negative chronic hepatitis B. N Engl J Med 2006;354:1011-1020.

10. Sherman M, Yurdaydin C, Sollano J, et al. Entecavir for treatment of lamivudine-refractory, $\mathrm{HBeAg}$-positive chronic hepatitis B. Gastroenterology 2006;130:2039-2049.

11. Bristol-Myers Squibb Company. Entecavir (BMS-200475): Antiviral Drugs Advisory Committee (AVDAC) briefing document [Internet]. Silver Spring (MD): Food and Drug Administration, 2005 [cited 2017 Sep 5]. Available from: https://www.fda.gov/ohrms/dockets/ac/05/briefing/2005-4094B1_01_BristolMyersSquibb-entecavir.pdf.

12. National Institutes of Health (NIH). Study of entecavir in patients with chronic hepatitis B virus infection [Internet]. Bethesda (MD): NIH, c2015 [cited 2017 Sep 5]. Available from: http://clinicaltrials.gov/ct2/show/NCToo388674.

13. Hou JL, Jia JD, Wei L, et al. Efficacy and safety of entecavir treatment in a heterogeneous CHB population from a 'real-world' clinical practice setting in China. J Viral Hepat 2013;20:811-820.
14. Mazzucco CE, Hamatake RK, Colonno RJ, Tenney DJ. Entecavir for treatment of hepatitis B virus displays no in vitro mitochondrial toxicity or DNA polymerase gamma inhibition. Antimicrob Agents Chemother 2008;52:598605 .

15. Liaw YF, Raptopoulou-Gigi M, Cheinquer H, et al. Efficacy and safety of entecavir versus adefovir in chronic hepatitis B patients with hepatic decompensation: a randomized, open-label study. Hepatology 2011;54:91-100.

16. Shim JH, Lee HC, Kim KM, et al. Efficacy of entecavir in treatment-naïve patients with hepatitis B virus-related decompensated cirrhosis. J Hepatol 2010;52:176-182.

17. Zoutendijk R, Reijnders JG, Brown A, et al. Entecavir treatment for chronic hepatitis B: adaptation is not needed for the majority of naive patients with a partial virological response. Hepatology 2011;54:443-451.

18. Manns MP, Akarca US, Chang TT, et al. Long-term safety and tolerability of entecavir in patients with chronic hepatitis B in the rollover study ETV-901. Expert Opin Drug Saf 2012;11:361-368.

19. Tenney DJ, Pokornowski KA, Rose RE, et al. 20 Entecavir maintains a high genetic barrier to HBV resistance through 6 years in naive patients. J Hepatol 2009;50(Suppl 1):S10.

20. Tenney DJ, Rose RE, Baldick CJ, et al. Long-term monitoring shows hepatitis B virus resistance to entecavir in nucleoside-naive patients is rare through 5 years of therapy. Hepatology 2009;49:1503-1514. 